\title{
Identificação do paciente em pronto-socorro de hospitais universitários: estudo
}

\section{transversal}

\author{
Patient identification in the emergency room of university hospitals: a cross-sectional study \\ Identificación de pacientes en urgencias de hospitales universitarios: estudio transversal
}

Recebido: 11/01/2021 | Revisado: 11/01/2021 | Aceito: 12/01/2021 | Publicado: 13/01/2021

\author{
Suelen Cristina Zandonadi Bernal \\ ORCID: https://orcid.org/0000-0002-1327-9261 \\ Universidade Estadual de Maringá, Brasil \\ E-mail: suelenbernal_85@hotamil.com \\ Daiane Cortez Raimondi \\ ORCID: https://orcid.org/0000-0002-7143-3706 \\ Universidade Estadual de Maringá, Brasil \\ E-mail: daianetcortez@gmail.com \\ Greice Westphal \\ ORCID: https://orcid.org/0000-0001-9107-0108 \\ Universidade Estadual de Maringá, Brasil \\ E-mail: greicewes@gmail.com \\ Nelson Nardo Junior \\ ORCID: https://orcid.org/0000-0002-6862-7868 \\ Universidade Estadual de Maringá, Brasil \\ E-mail: nnjunior@uem.br \\ Carlos Alexandre Molena Fernandes \\ ORCID: https://orcid.org/0000-0002-4019-8379 \\ Universidade Estadual de Maringá, Brasil \\ E-mail: carlosmolena126@gmail.com \\ Laura Misue Matsuda \\ ORCID: https://orcid.org/0000-0002-4280-7203 \\ Universidade Estadual de Maringá, Brasil \\ E-mail: lauramisuem@gmail.com
}

\begin{abstract}
Resumo
Objetivos: Determinar a prevalência de identificação do paciente em unidades de pronto-socorro, e verificar a adesão da equipe multiprofissional de unidades de pronto-socorro à conferência de dados de identificação do paciente. Métodos: Pesquisa descritiva, observacional, de abordagem quantitativa e delineamento transversal, realizada em prontos-socorros de três hospitais universitários, públicos, entre julho e setembro/2017. A população constituiu-se de 240 pacientes que permaneceram mais de 24 horas nessa unidade. Resultados: Constatou-se identificação com pulseira em $87,1 \%$ dos pacientes e prevalência de $48,3 \%$ na adesão da equipe de saúde à conferência dos dados de identificação do paciente. Quando comparados os hospitais, a adesão à conferência de dados foi de: $\mathrm{HA}=27,1 \%$; HB $=40,3 \% \mathrm{e} ; \mathrm{HC}=77,3 \%$. Conclusão: Nos serviços investigados, a identificação do paciente não era realizada de forma sistemática, especialmente na etapa de checagem dos dados antes da realização de procedimentos.
\end{abstract}

Palavras-chave: Sistemas de identificação de pacientes; Segurança do paciente; Serviços médicos de emergência.

\begin{abstract}
Objectives: To determine the prevalence of patient identification in emergency room units, and to verify the adherence of the multidisciplinary team of emergency room units to the patient identification data conference. Methods: Descriptive, observational, quantitative approach and cross-sectional research, carried out in emergency rooms at three public university hospitals, between July and September / 2017. The population consisted of 240 patients who remained in this unit for more than 24 hours. Results: Identification with a bracelet was found in $87.1 \%$ of the patients and a prevalence of $48.3 \%$ in the adherence of the health team to the verification of patient identification data. When comparing hospitals, adherence to the data conference was: $\mathrm{HA}=27.1 \% ; \mathrm{HB}=40.3 \%$ and; $\mathrm{HC}=77.3 \%$. Conclusion: In the services investigated, patient identification was not carried out systematically, especially in the step of checking the data before performing procedures.
\end{abstract}

Keywords: Patient identification systems; Patient safety; Emergency medical services.

\section{Resumen}

Objetivos: Determinar la prevalencia de identificación de pacientes en las unidades de urgencias y verificar la adherencia del equipo multidisciplinario de las unidades de urgencias a la conferencia de datos de identificación de 
pacientes. Métodos: Abordaje descriptivo, observacional, cuantitativo e investigación transversal, realizada en salas de emergencia de tres hospitales universitarios públicos, entre julio y septiembre / 2017. La población estuvo conformada por 240 pacientes que permanecieron en esta unidad por más de 24 horas. Resultados: Se encontró identificación con brazalete en el $87,1 \%$ de los pacientes y una prevalencia del 48,3\% en la adherencia del equipo de salud a la verificación de los datos de identificación del paciente. Al comparar hospitales, la adherencia a la conferencia de datos fue: $\mathrm{HA}=27,1 \% ; \mathrm{HB}=40,3 \%$ y; $\mathrm{HC}=77,3 \%$. Conclusión: En los servicios investigados, la identificación de los pacientes no se realizó de manera sistemática, especialmente en el paso de verificación de los datos antes de realizar los procedimientos.

Palabras clave: Sistemas de identificación de pacientes; Seguridad del paciente; Servicios médicos de emergencia.

\section{Introdução}

No sentido de maximizar o cuidado seguro, a Organização Mundial da Saúde (OMS) criou no ano de 2004 a Aliança Mundial para a Segurança do Paciente, com finalidade de divulgar e disseminar estratégias de boas práticas assistenciais, e estimular a cultura de segurança do paciente (WHO, 2008, 2012).

O Brasil, membro da referida Aliança, lançou em 2013 o Programa Nacional de Segurança do Paciente (PNSP)(Brasil, 2013) com vistas à melhoria da cultura de segurança do paciente e ao cuidado mais seguro, livre de eventos adversos que possam comprometer a saúde dos pacientes (Urbanetto \& Gerhardt, 2013). Para tal, as ações de segurança foram preconizadas por meio de seis Protocolos Básicos de Segurança do Paciente: Prevenção de úlcera por pressão; Segurança na prescrição, uso e administração de medicamentos; Cirurgia segura; Prática de higienização das mãos em serviço de saúde; Prevenção de quedas e; Identificação do paciente (Brasil, 2013).

Considerada de extrema relevância em instituições de saúde, a identificação é fator prioritário na segurança do paciente, pois tem como principal finalidade garantir que o procedimento/cuidado seja executado no paciente certo, evitandose eventos adversos no processo de cuidado (Brasil, 2013; Hoffmeister \& Moura, 2015).

A incongruência e/ou a falta de identificação do paciente podem ocasionar graves eventos adversos, tais como: erros de medicação, erros na administração de hemocomponentes, erros na entrega de diagnósticos, procedimentos realizados em pacientes inadequados e/ou locais errados da região corpórea, entrega de recém-nascidos a famílias erradas, dentre outros (WHO, 2008).

Na Europa, a European Federation of Clinical Chemistry and Laboratory Medicine Working Group for the Preanalytical Phase (EFLM WG-PRE) conduziu um estudo observacional em 12 países com a finalidade de identificar as áreas mais críticas referentes à segurança do paciente na coleta e armazenamento de amostras de sangue. A pesquisa apontou que a identificação do paciente se situa entre as áreas mais críticas, necessitando de melhora imediata, uma vez que o processo de identificação do paciente em tubos de amostra de sangue é uma barreira de segurança essencial para evitar a confusão da identidade do paciente (Simundic et al., 2015).

A identificação do paciente é condição primordial no processo de atendimento à saúde, haja vista o grande número de procedimentos complexos realizados nos pacientes, especialmente no setor de pronto-socorro (PS), o qual possui peculiaridades que maximizam os riscos de incidentes relacionados ao cuidado/atendimento (Zanelatto \& Dal Pai, 2010). Neste contexto, uma revisão sistemática realizada no ano de 2013, em serviços de emergência, com finalidade de estimar a prevalência dos EA, apontou variação de $0,2 \%$ a $6 \%$ na proporção de pacientes que sofreram EA relacionado ao cuidado, e evitabilidade entre $36 \%$ a $71 \%$ (Stang et al., 2013).

Para minimizar a ocorrência de erros associadas à identificação do paciente, a OMS propôs medidas normativas, tais como: Uso de pulseira desde a admissão até a alta (a qual deve ser conferida antes da realização de qualquer procedimento); estabelecimento de protocolos institucionais; e envolvimento do paciente/família no processo de identificação (WHO, 2003).

Considerando que a identificação do paciente é pedra angular para o cuidado seguro, questiona-se: Será que os 
pacientes atendidos na unidade de pronto-socorro de três instituições paranaenses são identificados? Antes de realizar qualquer procedimento, a equipe de saúde que atua em PS confere os dados da pulseira de identificação do paciente? Para responder a essas perguntas, o presente estudo teve como objetivos: determinar a prevalência de identificação do paciente em unidades de OS, e verificar a adesão da equipe multiprofissional de unidades de PS à conferência dos dados da pulseira de identificação do paciente.

\section{Metodologia}

Estudo descritivo, observacional, de abordagem quantitativa e delineamento transversal, realizado em três Hospitais Universitários da região Sul do Brasil, representados pelas siglas HA, HB e HC.

Utilizou-se amostragem não probabilística, cujos participantes foram selecionados por conveniência. O tamanho da amostra foi calculado a partir do número de internações do primeiro trimestre do ano de 2016, por estabelecimento de saúde. As informações foram obtidas do site DATASUS. O tamanho da amostra foi de 240 indivíduos, assim distribuídos: 61 do HA; 91 do $\mathrm{HB}$ e 88 do HC.

Os participantes foram todos os pacientes com tempo de permanência igual ou superior há $24 \mathrm{~h}$ no PS e em condições de verbalizar sobre o assunto questionado. Esse quesito foi verificado por meio da aplicação da Escala de Glasgow, que avalia o nível de consciência do entrevistado.

As entrevistas foram realizadas em pacientes com Glasgow entre 13 e 15 pontos, os quais são obtidos através da observação do paciente atribuindo um valor a cada parâmetro observado (abertura ocular, resposta verbal e resposta motora), sendo que a soma dos pontos atribuídos a cada parâmetro constitui o resultado da escala (Teasdale Graham et al., 2014).

A coleta de dados foi realizada por meio de observação e entrevista estruturada, no período de julho a setembro de 2017, utilizando-se um formulário de entrevista, composto pelas seguintes variáveis: perfil sociodemográfico, tipo de identificação utilizada, conferência à identificação do paciente pela equipe multiprofissional.

Os dados foram agrupados em banco de dados no Excel 2010 (Microsoft Inc.) e, posteriormente, analisados no programa SPSS (Statistical Package for the Social Sciences) (Andy Field, 2009), versão 20.0. Para comparar possíveis diferenças entre os hospitais, foi aplicado o teste qui-quadrado para as variáveis categóricas. Para todas as análises realizadas, adotou-se nível de significância de $\alpha=0,05$. As variáveis categóricas foram inseridas em tabelas de contingência e gráficos, contendo frequências absolutas (n) e relativas (\%).

Esta pesquisa atendeu aos princípios éticos da Resolução n466/12, do Conselho Nacional de Saúde e está registrado

Comitê Permanente de Ética em Pesquisas Envolvendo Seres Humanos (COPEP), da Universidade Estadual de Maringá (UEM), sob parecer nº 2.144.505, CAAE 65459617.7.0000.0104, JUN/2017.

\section{Resultados}

Na Tabela 1, constam os dados sócio demográficos da amostra. 
Research, Society and Development, v. 10, n. 1, e28810111864, 2021

(CC BY 4.0) | ISSN 2525-3409 | DOI: http://dx.doi.org/10.33448/rsd-v10i1.11864

Tabela 1. Caracterização sócio demográfica de pacientes atendidos em três prontos-socorros. Sul do Brasil, 2017.

\begin{tabular}{|c|c|c|c|c|c|c|c|c|}
\hline \multicolumn{9}{|c|}{ Hospitais } \\
\hline Variáveis & \multicolumn{2}{|c|}{$A(n=61)$} & \multicolumn{2}{|c|}{$B(n=91)$} & \multicolumn{2}{|c|}{$C(n=88)$} & \multicolumn{2}{|c|}{ Total $(n=240)$} \\
\hline SEXO & $\mathrm{F}$ & $(\%)$ & $\mathrm{f}$ & $(\%)$ & $\mathrm{F}$ & $(\%)$ & $\mathrm{F}$ & $(\%)$ \\
\hline Masculino & 34 & $(55,0)$ & 43 & $(47,3)$ & 38 & $(43,2)$ & 115 & $(47,9)$ \\
\hline Feminino & 27 & $(44,3)$ & 48 & $(52,7)$ & 50 & $(56,8)$ & 125 & $(52,1)$ \\
\hline \multicolumn{9}{|l|}{ FAIXA ETÁRIA } \\
\hline$<40$ anos & 25 & $(40,9)$ & 46 & $(50,5)$ & 25 & $(28,4)$ & 96 & $(40,0)$ \\
\hline $40-59$ anos & 21 & $(34,4)$ & 28 & $(30,8)$ & 21 & $(23,9)$ & 70 & $(29,2)$ \\
\hline$\geq 60$ anos & 15 & $(24,7)$ & 17 & $(18,7)$ & 42 & $(47,7)$ & 74 & $(30,8)$ \\
\hline \multicolumn{9}{|l|}{ ESCOLARIDADE } \\
\hline Até 4 anos & 14 & $(23,0)$ & 14 & $(15,4)$ & 35 & $(39,8)$ & 63 & $(26,2)$ \\
\hline 5 a 10 anos & 25 & $(41,0)$ & 38 & $(41,8)$ & 22 & $(25,0)$ & 85 & $(35,4)$ \\
\hline 11 anos ou mais & 22 & $(36,0)$ & 39 & $(42,8)$ & 31 & $(35,2)$ & 92 & $(38,4)$ \\
\hline
\end{tabular}

No Gráfico 1, constam dados de prevalência de identificação do paciente nos três PS. Nota-se que a prevalência de identificação, considerando o total da amostra, foi de $87,1 \%(n=209)$.

Gráfico 1. Prevalência de identificação do paciente em prontos-socorros. Sul do Brasil, 2017.

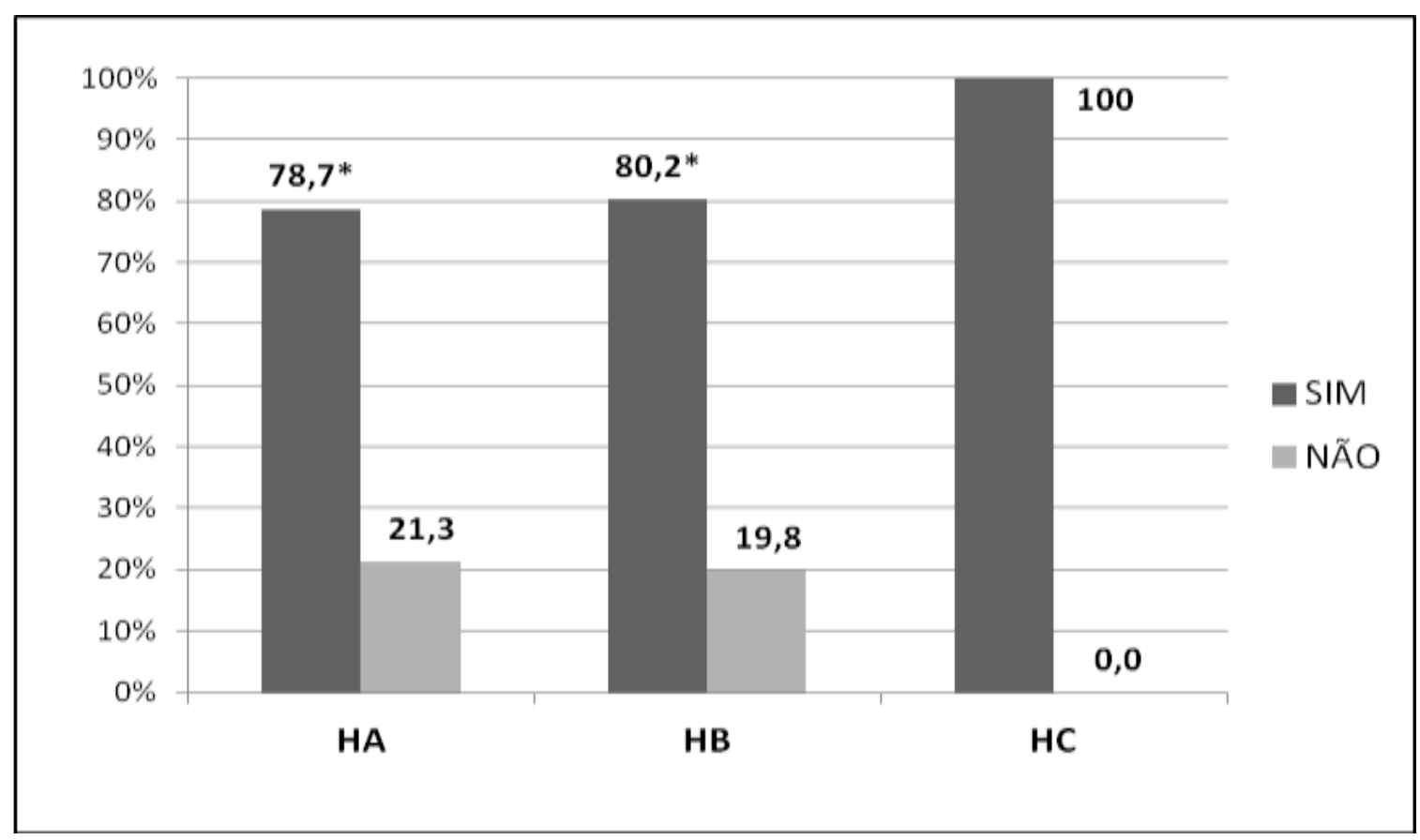

*Diferença significativa em relação ao HC para p<0,05 (Qui-quadrado). Fonte: Autores.

No Gráfico 2, constam dados da prevalência de adesão à conferência dos dados de identificação do paciente pelos profissionais de saúde, no três PS investigados. Quando observada a amostra total, a adesão à conferência de identificação do paciente pelos profissionais de saúde foi de 50,8\% $(n=122)$. 
Gráfico 2. Prevalência de adesão à conferência dos dados de identificação de pacientes em prontos-socorros. Sul do Brasil, 2017.

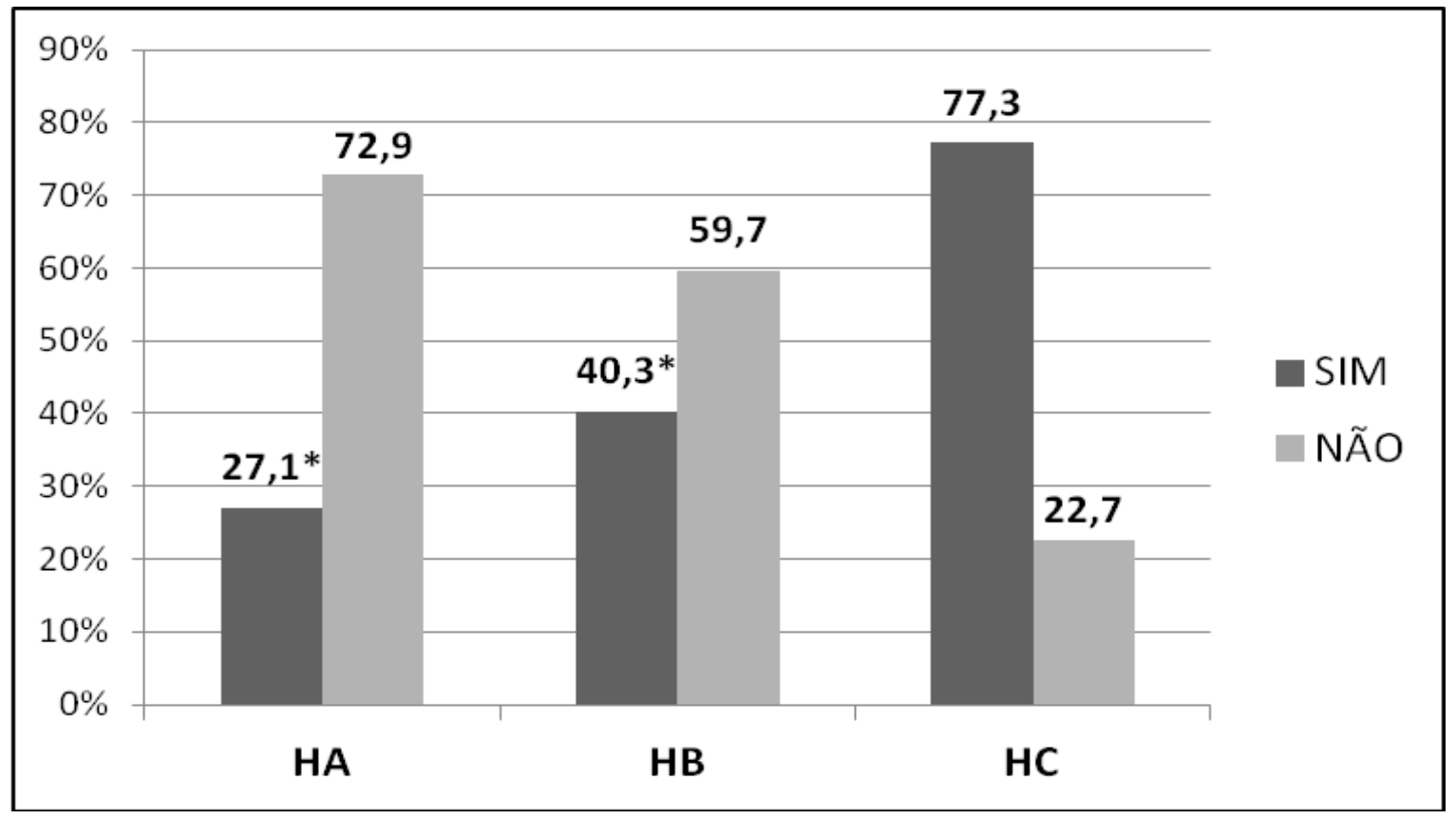

*Diferença significativa em relação ao $\mathrm{HC}$ para p<0,05 (Qui-quadrado). Fonte: Autores.

\section{Discussão}

Conforme se evidencia na Tabela 1, dentre os 240 participantes, 125 (52,1\%) eram do sexo feminino. Este perfil da população corrobora com um estudo realizado no município do estado de São Paulo, o qual constatou que 58\% das pessoas que procuravam o serviço de pronto-socorro eram do sexo feminino (Oliveira et al., 2011). Em relação à faixa etária que mais utilizou a unidade, $144(60 \%)$ tinham idade acima de 40 anos, e apenas 177 (73,8\%) haviam frequentado escola por mais de cinco anos.

Neste estudo, constatou-se que $209(87,1 \%)$ pacientes apresentavam identificação por meio de pulseira. Embora essa prevalência aparente ser um resultado positivo (mais de 50\%), o número de pacientes identificados deveria ser o mais próximo possível de $100 \%$ porque falhas na identificação do paciente podem levar a eventos catastróficos (Hoffmeister \& Moura, 2015).

Ao comparar os resultados da prevalência de pacientes identificados entre os PS (Gráfico 1), os números indicam que, no HA, 21,3\% dos pacientes não estavam identificados; no Hospital B, a prevalência de pacientes não identificados era de 19,8\%, e, no HC, não houve casos de pacientes sem identificação. Apesar de a identificação do paciente ser uma estratégia inerente ao cuidado seguro (Brasil, 2013), observa-se que, dos três PS investigados, dois apresentam resultados que podem ser considerados alarmantes. Isso porque um estudo de Coorte, realizado nos Emirados Árabes, em pacientes do internamento, constatou que a ausência de pulseiras corresponde a mais da metade dos eventos adversos relacionados à identificação do paciente (Dhatt et al., 2011).

Segundo a WHO (2008), a primeira meta estabelecida para a segurança do paciente é sua identificação correta e, para que isso aconteça, é preciso que as instituições adotem formas para identificar seus pacientes, a exemplo da pulseira de identificação, contendo, no mínimo, dois elementos identificadores. Além disso, devem-se estabelecer estratégias para que o 
seu uso seja assegurado e a sua conferência sejam permanentes antes da realização de procedimentos (Brasil, 2013; Smith et al., 2011; WHO, 2008).

Com relação aos dados referentes à adesão à verificação da identificação do paciente, neste estudo, a prevalência de não adesão correspondeu a mais da metade do total da amostra (51,9\%). Dado semelhante foi observado num estudo realizado em um hospital universitário, nas unidades de internação adulta e pediátrica da cidade de Porto Alegre, com objetivo de verificar a adesão dos profissionais à verificação da pulseira de identificação. Nesse estudo, observou-se $57,1 \%$ de prevalência de não adesão (Prade Hemesath et al., 2015). Já no Reino Unido, estudo que teve o objeto de analisar o processo de identificação do paciente constatou que as pulseiras de identificação não eram checadas pelos profissionais de saúde antes da realização de procedimentos, demonstrando que esta etapa do processo de identificação ainda é negligenciada (Smith et al., 2011).

No que tange a prevalência de adesão à conferência de identificação do paciente, ao comparar as três instituições (Gráfico 2), constata-se diferença significativa de HA e HB em relação ao HC, representada respectivamente por 27,1\%, 40,3\% e 77,3\% de adesão à conferência dos dados de identificação do paciente. Nesse aspecto, o Hospital A apresentou prevalência significativamente superior de não adesão à conferência de identificação (72,9\%), em relação aos demais hospitais, com destaque ao Hospital C, que apresentou o menor percentual de não adesão $(22,7 \%)$.

O resultado do Hospital C certamente é consequência do serviço denominado Assessoria de Controle de Qualidade da Assistência de Enfermagem, liderado por um enfermeiro e implantado há quase 20 anos na instituição. Além disso, nesse mesmo hospital, o Núcleo de Segurança do Paciente (NSP) foi implantando há mais tempo que nos demais. No HA, por exemplo, até o momento, não existe serviço específico da Qualidade e o processo de implantação do NSP ainda se encontra em fase inicial. Já no HB, há um escritório de Qualidade e a implantação do NSP se encontra em fase intermediária.

Apesar da intensificação de medidas para otimizar o processo de identificação de pacientes, um estudo inglês revela que, apesar de a pulseira de identificação ser amplamente recomendada, a produção cientifica sobre a temática é incipiente e o seu resultado não tem sido utilizado adequadamente para prevenir eventos adversos, devido ao desconhecimento da sua importância pelos profissionais de saúde (Smith et al., 2011).

Ressalta-se que, no processo de atendimento à saúde, para reduzir eventos adversos relacionados à identificação do paciente, a pulseira é considerada o meio mais efetivo, porém, além do seu uso, é necessário que todos os profissionais realizem a checagem dos dados de identificação, confirmando os identificadores preconizados, antes de realizar quaisquer procedimentos no paciente.

\section{Conclusão}

Concluiu-se que, em dois hospitais (HA e HB), cerca de $20 \%$ dos pacientes investigados não apresentavam identificação. Por outro lado, o HC apresentou resultado satisfatório, uma vez que todos os pacientes estavam adequadamente identificados.

No que tange a verificação dos dados de identificação dos pacientes, constatou-se que, nas unidades de pronto-socorro investigadas, a checagem dos dados pelos profissionais de saúde não é realizada sistematicamente $(\mathrm{HA}=72,9 \%$; $\mathrm{HB}=59,7 \%$ e $\mathrm{HC}=22,7 \%$ ).

Em que pese a importância dos resultados deste estudo, depreende-se que os mesmos apresentam limitações por terem sido levantados por meio de delineamento transversal único, e, também, por ter sido realizado somente em Prontos Socorros hospitalares. Com isso, sugerem-se estudos de Coorte, em diferentes setores e instituições de saúde, para acompanhamento do 
Research, Society and Development, v. 10, n. 1, e28810111864, 2021 (CC BY 4.0) | ISSN 2525-3409 | DOI: http://dx.doi.org/10.33448/rsd-v10i1.11864

processo de identificação, que sejam capazes de fornecer informações com mais evidências ao fomento da segurança do paciente.

\section{Referências}

Andy Field. (2009). Discovering Statistics using SPSS Statistics. SAGE Publications, 66, 822. http://www.amazon.com/Discovering-Statistics-using-IBMSPSS/dp/1446249182

Brasil, M. da S. (2013). Portaria $n^{\circ}$. 529, de 1 de abril de 2013. Institui o Programa Nacional de Segurança do Paciente (PNSP). http://bvsms.saude.gov.br/bvs/saudelegis/gm/2013/prt0529_01_04_2013.html

Brasil, M. da S. (2013). Protocolo de Identificação do Paciente. http://www.anvisa.gov.br/hotsite/segurancadopaciente/documentos/julho/Protocolo Identificação do Paciente.pdf

Brasil, M. da S. (2013). Relatório do seminário nacional para implantação do programa nacional de segurança do paciente. http://www.anvisa.gov.br/hotsite/segurancadopaciente/Documentos/RelatorioSeminarioNacionalImplantacaodoProgramaNacionaldeSegurancadoPacienteout2 013.pdf

Dhatt, G. S., Damir, H. A., Matarelli, S., Sankaranarayanan, K., \& James, D. M. (2011). Patient safety: Patient identification wristband errors. Clinical Chemistry and Laboratory Medicine, 49(5), 927-929. https://doi.org/10.1515/CCLM.2011.129

Hoffmeister, L. V., \& Moura, G. M. S. S. de. (2015). Use of identification wristbands among patients receiving inpatient treatment in a teaching hospital. Revista Latino-Americana de Enfermagem, 23(1), 36-43. https://doi.org/10.1590/0104-1169.0144.2522

Oliveira, G. N., Silva, M. de F. N., Araujo, I. E. M., \& Carvalho-Filho, M. A. (2011). Profile of the Population Cared for in a Referral Emergency Unit. Revista Latino-Americana de Enfermagem, 19(3), 548-556. https://doi.org/10.1590/s0104-11692011000300014

Prade Hemesath, M., Barreto dos Santos, H., Schroder Torelly, E., Bolzan Motta, M., Silveira Pasin, S., \& Muller de Magalhães, A. (2015). Avaliação e gestão da adesão dos profissionais à verificação da identificação do paciente. Revista Acreditação, 5(9), 45-54.

Simundic, A. M., Church, S., Cornes, M. P., Grankvist, K., Lippi, G., Nybo, M., Nikolac, N., Van Dongen-Lases, E., Eker, P., Kovalevskaya, S., Kristensen, G. B. B., Sprongl, L., \& Sumarac, Z. (2015). Compliance of blood sampling procedures with the CLSI H3-A6 guidelines: An observational study by the European Federation of Clinical Chemistry and Laboratory Medicine (EFLM) working group for the preanalytical phase (WG-PRE). Clinical Chemistry and Laboratory Medicine, 53(9), 1321-1331. https://doi.org/10.1515/cclm-2014-1053

Smith, A. F., Casey, K., Wilson, J., \& Fischbacher-Smith, D. (2011). Wristbands as aids to reduce misidentification: An ethnographically guided task analysis. International Journal for Quality in Health Care, 23(5), 590-599. https://doi.org/10.1093/intqhc/mzr045

Stang, A. S., Wingert, A. S., Hartling, L., \& Plint, A. C. (2013). Adverse Events Related to Emergency Department Care: A Systematic Review. PLoS ONE, 8(9). https://doi.org/10.1371/journal.pone.0074214

Teasdale Graham, A, D., Brennan, P., McElhinney, E., \& Mckinnon, L. (2014). Forty years on: updating the Glasgow Coma Scale. Nursing Times, 110(42), $12-16$.

Urbanetto, J. de S., \& Gerhardt, L. M. (2013). Segurança do paciente na tríade assistência ensino pesquisa. Revista Gaúcha de Enfermagem, 34(3), 8-9. https://doi.org/10.1590/s1983-14472013000300001

WHO, W. H. O. (2003). Patient Identification: The Foundation for a Culture of Patient Safety. Journal of Nursing Care Quality, 18(1), 73-79. https://doi.org/10.1097/00001786-200301000-00010

WHO, World. Health. Organization. (2008). Summary of the evidence on patient safety: implications for research. In A. Jha (Org.), Ashish Jha (p. 136).

WHO, World. Health. Organization (2012). Patient safety research: a guide for developing training programmes. In WHO (Org.), WHO.

Zanelatto, D. M., \& Dal Pai, D. (2010). Práticas de acolhimento no serviço de emergência: a perspectiva dos profissionais de enfermagem. Ciência, Cuidado e Saúde, 9(2). https://doi.org/10.4025/cienccuidsaude.v9i2.9390 